\title{
The Flow of Newtonian and power law fluids in elastic tubes
}

\author{
Taha Sochi
}

\begin{abstract}
University College London, Department of Physics \& Astronomy, Gower Street, London, WC1E 6BT
\end{abstract}
Email: t.sochi@ucl.ac.uk.

\begin{abstract}
We derive analytical expressions for the flow of Newtonian and power law fluids in elastic circularly-symmetric tubes based on a lubrication approximation where the flow velocity profile at each cross section is assumed to have its axially-dependent characteristic shape for the given rheology and cross sectional size. Two pressure-area constitutive elastic relations for the tube elastic response are used in these derivations. We demonstrate the validity of the derived equations by observing qualitatively correct trends in general and quantitatively valid asymptotic convergence to limiting cases. The Newtonian formulae are compared to similar formulae derived previously from a one-dimensional version of the Navier-Stokes equations.
\end{abstract}

Keywords: fluid dynamics; elastic tube; Newtonian fluid; power law fluid; 1D Navier-Stokes; lubrication approximation.

\section{Introduction}

The flow of Newtonian and non-Newtonian fluids in distensible conduits of elastic or viscoelastic nature is common in many biological and industrial systems. Some examples are the flow of biological fluids such as blood in the vessels and porous tissues of most organisms including human, the transportation of drainage extracts, and the delivery of various liquid products in the oil and food industries.

A number of attempts have been made in the past to model the flow of fluids in distensible conduits; most of which are restricted to Newtonian fluids and relatively few considered non-Newtonian rheologies. An example of the former is the widely used one-dimensional (1D) Navier-Stokes flow model in deformable tubes [1-6], while an example of the latter is the attempt of Vajravelu et al [7] to model the flow of Herschel-Bulkley fluids in elastic tubes. Other attempts have also been made to deal with more special cases of non-Newtonian flow using mainly numerical methods $[8,9]$.

Although the 1D Navier-Stokes distensible flow model is widely used in the flow simulations in single distensible tubes and networks of interconnected distensible 
tubes especially in the hemodynamic studies, it has a number of limitations. One of these limitations is its restriction to the Newtonian flow, on which the NavierStokes momentum equation is based, and hence to accommodate non-Newtonian rheologies, the use of approximations or employing different models, if they exist, is required. Another limitation is the relatively large number of parameters (e.g. $\alpha, \kappa$, $\rho$, etc.) that define the $1 \mathrm{D}$ model; which make it difficult to choose proper numerical values for these parameters in practical situations without considerable amount of experimental and observational work, and hence some ambiguity or arbitrariness may be attached to the assumptions and results of this model. This has been demonstrated in [10] where the results of the 1D Navier-Stokes flow model can vary considerably on varying these parameters; and hence the model may loose its predictive value due to large uncertainties in the results as a consequence of uncertainties in the input parameters.

Concerning the non-Newtonian case, the approaches developed to deal with various non-Newtonian rheologies in distensible conduits face serious analytical and numerical difficulties; hence hindering their development and usage and limiting their formulation and application to rather special cases with considerable approximations. A quick review to the existing literature will reveal that limited progress has been made on most aspects of the non-Newtonian flow in deformable conduits. There are many gaps in the research literature on this subject where no attempts have been made to investigate some commonplace problems.

Based on this assessment to the existing Newtonian and non-Newtonian flow models, it can be concluded that it is highly desirable to have flow models with comparatively few parameters that can describe both Newtonian and non-Newtonian rheologies even if they are developed by the employment of simpler approaches. Although such models may seem idealized, they can be used in many practical situations; moreover, they can serve as prototypes for the development of more sophisticated models.

In the present paper, we try to develop one such model for the Newtonian and power law fluids using two pressure-area elastic relations to describe the tube distensibility. We use a lubrication approximation approach based on assuming a fluid-specific velocity profile that varies with the size of the cross sectional area which can be assumed to vary smoothly in the axial direction for the investigated case of laminar flow through distensible conduits. We partially validate the derived analytical expressions by observing a number of sensible trends mostly related to convergence to limiting cases. We also propose the use of these expressions in 
conjunction with the previously proposed [11] residual-based pore-scale network modeling to simulate the flow of Newtonian and power law fluids in networks of interconnected distensible tubes that can also be used as models for porous media.

Extending the method to rheologies other than Newtonian and power law fluids and to conduits of distensible nature other than the two simple pressure-area elastic models is a possibility especially if numerical, as well as analytical, methods are considered. Also, broadening the proposed method to include conduit geometries other than the regular cylindrical tube with a constant cross sectional area in the axial direction (e.g. conduits of elliptically-shaped cross sections or of convergingdiverging nature) may also be possible; although we will not consider any of these extensions in the present paper.

\section{Pressure-Area Relations}

There are many constitutive models that correlate the local pressure to the local cross sectional area in distensible tubes; these models include elastic and viscoelastic mechanical response, and linear and nonlinear correlation, as well as many other more specific variations. In this paper we use two elastic models to demonstrate the use of the proposed method.

One of these is a simple model in which a linear pressure-area correlation is assumed, that is

$$
p=\gamma\left(A-A_{o}\right)
$$

where $\gamma$ is the proportionality factor which is a measure for the stiffness of the tube wall, $A$ is the tube cross sectional area at the actual pressure $p$ as opposite to the reference pressure which, for the sake of convenience without loss of generality, is assumed to be zero, and $A_{o}$ is the reference area corresponding to the reference pressure. The core of this model is that the locally defined axial pressure is proportional to the change in the local cross sectional area relative to its reference state, where, for all the cases considered in this paper, we assume $A \geq A_{o}$ to exclude the collapsible tube case.

The other pressure-area model is based on the proportionality between the local pressure and the change in the local radius relative to its reference state with a proportionality stiffness factor that is scaled by the reference area, i.e. 


$$
p=\frac{\beta}{A_{o}}\left(\sqrt{A}-\sqrt{A_{o}}\right)
$$

where $\beta$ is the tube stiffness factor.

In the next section we use these two pressure-area models to derive flow relations for Newtonian and power law fluids using the proposed lubrication method.

\section{Derivation}

In this section we derive analytical relations for the volumetric flow rate, $Q$, as a function of the pressure boundary conditions in distensible tubes for the Newtonian and power law rheologies using the two pressure-area elastic constitutive relations given in the preceding section. In these derivations we assume an incompressible, time-independent, laminar, fully developed flow at relatively low Reynolds numbers with minimal entry and exit edge effects. The impact of edge effects should be reduced for sufficiently long tubes in laminar flow conditions. We also assume that the elastic tube has a fixed circularly-shaped cross section with a constant cross sectional area in the axial direction under unstressed state conditions. Another assumption is that the tube has a constant length and hence any stretch in the axial direction under stressed state conditions is negligible. The vessel wall is normally assumed to be thin, homogeneous, isotropic, of constant thickness with linear distensibility and negligible compressibility [12-14]; and we follow suit. The essence of these conditions is that the same pressure-area relation applies at all cross sections producing an axi-symmetric change in the tube geometry.

\subsection{Newtonian Fluids}

For Newtonian fluids the rheology is given by the following stress-strain relation

$$
\tau=\mu \dot{\gamma}
$$

where $\tau$ is the shear stress, $\mu$ is the fluid dynamic viscosity, and $\dot{\gamma}$ is the rate of shear strain. Based on the Hagen-Poiseuille flow assumptions, the volumetric flow rate, $Q$, is correlated to the fluid dynamic viscosity, $\mu$, tube radius, $r$, and pressure gradient $\frac{d p}{d x}$ through the following relation

$$
Q=\frac{\pi r^{4}}{8 \mu} \frac{d p}{d x}=\frac{A^{2}}{8 \pi \mu} \frac{d p}{d x}
$$


where $x$ is the tube axial coordinate, $p$ is the pressure in the axial direction and $A$ is the cross sectional area. If we assume, as a consequence of the validity of the aforementioned assumption of flow profile dependency, that such a relation is valid at each cross section even for an elastic tube with variable cross sectional area that is subject to an axially-dependent pressure as long as the variation in the pressure, and hence the corresponding cross sectional area, is smooth, we then have the following relation at each cross section

$$
\frac{d p}{d x}=\frac{8 \pi \mu Q}{A^{2}}
$$

where $Q$ as a function of the axial coordinate, $x$, is constant due to the incompressibility and time-independent flow conditions.

Now, from the first $p$ - $A$ relation we have

$$
A=\frac{p}{\gamma}+A_{o}
$$

and hence from Equation 5 we obtain

$$
\frac{d p}{d x}=\frac{8 \pi \mu Q}{\left(\frac{p}{\gamma}+A_{o}\right)^{2}}
$$

which can be separated and integrated, i.e.

$$
\int\left(\frac{p}{\gamma}+A_{o}\right)^{2} d p=\int 8 \pi \mu Q d x
$$

to yield the following relation

$$
\frac{\left(p+\gamma A_{o}\right)^{3}}{3 \gamma^{2}}=8 \pi \mu Q x+C
$$

where $C$ is the constant of integration. From the inlet boundary condition, we have $p(x=0)=p_{\text {in }}$ where $p_{\text {in }}$ is the pressure at the tube inlet, and hence

$$
C=\frac{\left(p_{i n}+\gamma A_{o}\right)^{3}}{3 \gamma^{2}}
$$

that is

$$
Q=-\frac{\left(p_{i n}+\gamma A_{o}\right)^{3}-\left(p+\gamma A_{o}\right)^{3}}{24 \pi \mu \gamma^{2} x}
$$

where the minus sign indicates the fact that the flow is opposite in direction to 
the pressure gradient, i.e. $p_{\text {in }}>p_{\text {ou }}$ where $p_{\text {ou }}$ is the pressure at the tube outlet. Applying the second boundary condition at the outlet, i.e. $p(x=L)=p_{\text {ou }}$ where $L$ is the tube length, and dropping the minus sign as we are interested only in the magnitude of the flow rate since its direction is well known (i.e. from inlet to outlet), we obtain

$$
Q=\frac{\left(p_{i n}+\gamma A_{o}\right)^{3}-\left(p_{o u}+\gamma A_{o}\right)^{3}}{24 \pi \mu \gamma^{2} L}
$$

Following a similar procedure and using the second pressure-area relation, i.e. Equation 2, we obtain

$$
Q=\frac{\beta}{40 \pi \mu A_{o} L}\left[\left(\frac{A_{o}}{\beta} p_{\text {in }}+\sqrt{A_{o}}\right)^{5}-\left(\frac{A_{o}}{\beta} p_{\text {ou }}+\sqrt{A_{o}}\right)^{5}\right]
$$

\subsection{Power Law Fluids}

For power law fluids the stress-strain rheological relation is given by

$$
\tau=k \dot{\gamma}^{n}
$$

where $k$ is the power law consistency factor and $n$ is the flow behavior index. Based on the previously given assumptions, the following relation for the volumetric flow rate as a function of the fluid rheology, tube radius and pressure gradient can be derived $[15,16]$

$$
Q=\frac{\pi n}{3 n+1} \sqrt[n]{\frac{1}{2 k} \frac{d p}{d x}} r^{3+1 / n}=\frac{\pi n}{3 n+1} \sqrt[n]{\frac{1}{2 k} \frac{d p}{d x}}\left(\sqrt{\frac{A}{\pi}}\right)^{3+1 / n}
$$

which can be manipulated to give

$$
\frac{d p}{d x}=\frac{2 k \pi^{(n+1) / 2}(3 n+1)^{n} Q^{n}}{n^{n} A^{(3 n+1) / 2}}
$$

On using the first $p$ - $A$ relation to replace $A$ with $p$, separating the $p$ and $x$ variables, integrating and applying the two boundary conditions, as demonstrated in the Newtonian case, we obtain

$$
Q=\left(\frac{\gamma n^{n}\left[\left(\frac{p_{i n}}{\gamma}+A_{o}\right)^{3(n+1) / 2}-\left(\frac{p_{o u}}{\gamma}+A_{o}\right)^{3(n+1) / 2}\right]}{3 k \pi^{(n+1) / 2}(3 n+1)^{n}(n+1) L}\right)^{1 / n}
$$


Repeating the process with the use of the second $p$ - $A$ relation we obtain

$$
Q=\left(\frac{\beta n^{n}\left[\left(\frac{A_{o}}{\beta} p_{i n}+\sqrt{A_{o}}\right)^{(3 n+2)}-\left(\frac{A_{o}}{\beta} p_{o u}+\sqrt{A_{o}}\right)^{(3 n+2)}\right]}{2 k \pi^{(n+1) / 2}(3 n+1)^{n}(3 n+2) A_{o} L}\right)^{1 / n}
$$

\section{Validation}

We do not have an independent way, such as experimental data, to validate the derived formulae. However, we observe a number of sensible trends that can be regarded as partial verification:

- For both cases of Newtonian and power law fluids, we observe the convergence of the flow rate as obtained from the derived formulae to their rigid equivalents with increasing the tube stiffness, that is the convergence of Equations 12 and 13 to the Poiseuille flow for the Newtonian fluids, as given by Equation 4, and the convergence of Equations 17 and 18 to the power law flow, as given by Equation 15, for a tube with constant radius and hence constant pressure gradient in the axial direction. In Figures 1 and 2 we demonstrate this trend by a few examples from the Newtonian and power law fluids using the two pressure-area constitutive elastic relations.

- Another validation test is the convergence of the power law formulae for elastic tubes (Equations 17 and 18) to their corresponding Newtonian formulae (Equations 12 and 13) when $n=1.0$. In Figure 3 we demonstrate this using two examples: one from the first $p$ - $A$ elastic tube model (Equation 1), and the second from the second $p$ - $A$ elastic tube model (Equation 2). As seen, the Newtonian and power law formulae, which are derived independently using two different flow relations, produce identical results in this case as it should be. A consequence of this convergence is that in practical situations the Newtonian formulae are redundant as they can be obtained as a special case from the power law formulae.

- As a consequence of the previous two points, the power law formulae (Equations 17 and 18) converge to the rigid Poiseuille flow (Equation 4) when $n=1.0$ with high tube stiffness. This trend was verified in all the cases that were investigated. 
- There are several other qualitatively sensible trends that were verified in the newly derived formulae. These trends include shear thinning and shear thickening behaviors in the case of power law fluids, radius and pressure variation in the axial direction, and the magnitude of the flow and its connection to the average cross sectional area.

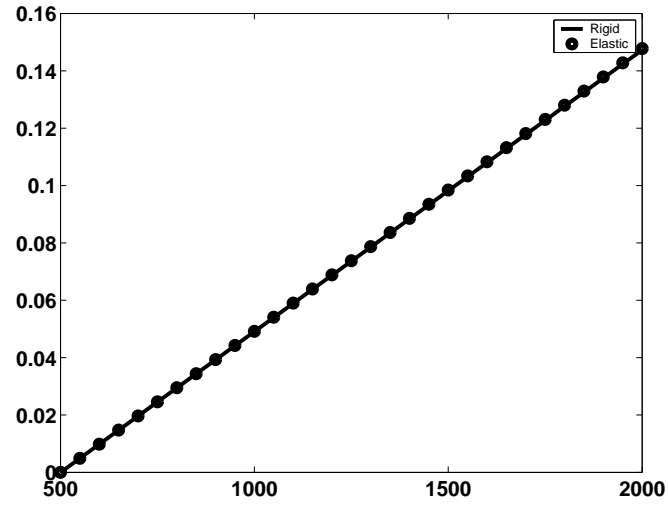

(a) Newtonian

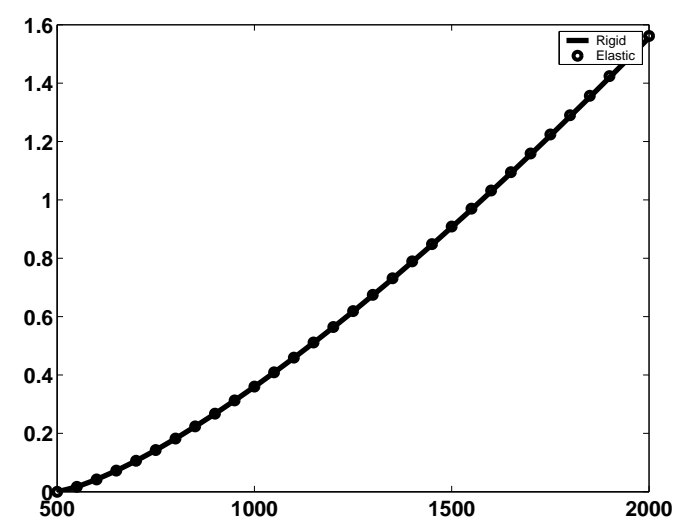

(b) Power law

Figure 1: Convergence of the elastic tube formulae (Equations 12 and 17) to their rigid equivalents (Equations 4 and 15) at high tube wall stiffness for the first $p$ - $A$ elastic tube model (Equation 1) (a) for a Newtonian fluid with $\mu=0.05$ Pa.s; and (b) for a shear thinning power law fluid with $k=0.05 \mathrm{~Pa}^{n} \mathrm{~s}^{n}$, and $n=0.75$. The tube parameters are: $\gamma=10^{8} \mathrm{~Pa}^{-2} \mathrm{~m}^{-2}, L=0.5 \mathrm{~m}$, and $R_{o}=0.05 \mathrm{~m}$. The vertical axis in the sub-figures represents the volumetric flow rate, $Q$, in $\mathrm{m}^{3} . \mathrm{s}^{-1}$ while the horizontal axis represents the inlet boundary pressure, $p_{i n}$, in $\mathrm{Pa}$. The outlet pressure is held constant at $p_{\text {ou }}=500 \mathrm{~Pa}$.

\section{Comparison to 1D Navier-Stokes Model}

In [17] two formulae based on the widely used 1D Navier-Stokes model for the flow of Newtonian fluids in elastic tubes were derived. These formulae, which are based on the first and second $p$ - $A$ relations respectively, are given by

$$
Q=\frac{L-\sqrt{L^{2}-4 \frac{\alpha}{\kappa} \ln \left(A_{\text {ou }} / A_{\text {in }}\right) \frac{\gamma}{3 \kappa \rho}\left(A_{\text {in }}^{3}-A_{\text {ou }}^{3}\right)}}{2 \frac{\alpha}{\kappa} \ln \left(A_{\text {ou }} / A_{\text {in }}\right)}
$$

and

$$
Q=\frac{-\kappa L+\sqrt{\kappa^{2} L^{2}-4 \alpha \ln \left(A_{\text {in }} / A_{\text {ou }}\right) \frac{\beta}{5 \rho A_{o}}\left(A_{\text {ou }}^{5 / 2}-A_{\text {in }}^{5 / 2}\right)}}{2 \alpha \ln \left(A_{\text {in }} / A_{\text {ou }}\right)}
$$




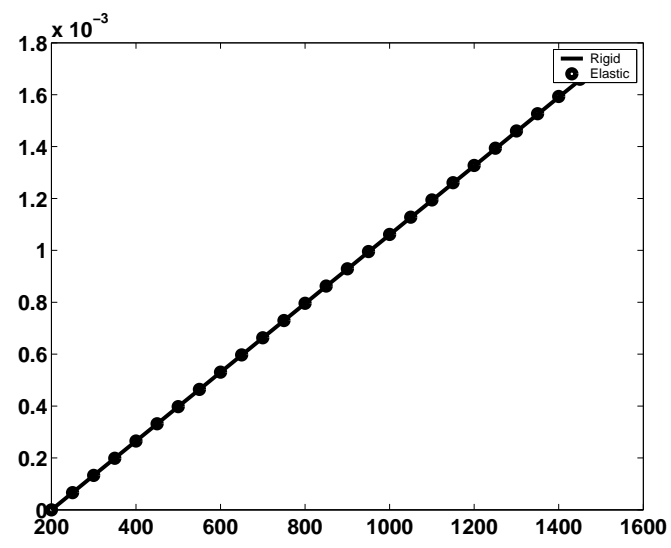

(a) Newtonian

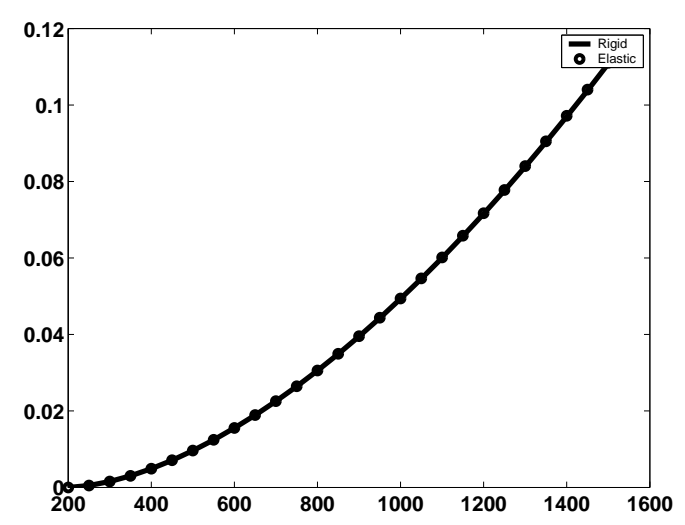

(b) Power law

Figure 2: Convergence of the elastic tube formulae (Equations 13 and 18) to their rigid equivalents (Equations 4 and 15) at high tube wall stiffness for the second $p$ - $A$ elastic tube model (Equation 2) (a) for a Newtonian fluid with $\mu=0.15$ Pa.s; and (b) for a shear thinning power law fluid with $k=0.15 \mathrm{~Pa} \cdot \mathrm{s}^{n}$, and $n=0.6$. The tube parameters are: $\beta=50000$ Pa.m, $L=0.1 \mathrm{~m}$, and $R_{o}=0.015 \mathrm{~m}$. The vertical axis in the sub-figures represents the volumetric flow rate, $Q$, in $\mathrm{m}^{3} . \mathrm{s}^{-1}$ while the horizontal axis represents the inlet boundary pressure, $p_{i n}$, in $\mathrm{Pa}$. The outlet pressure is held constant at $p_{\text {ou }}=200 \mathrm{~Pa}$.

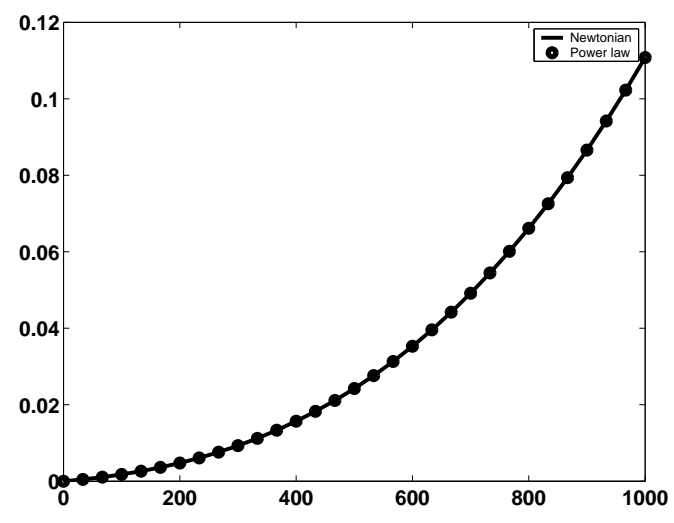

(a) First $p$ - $A$ model

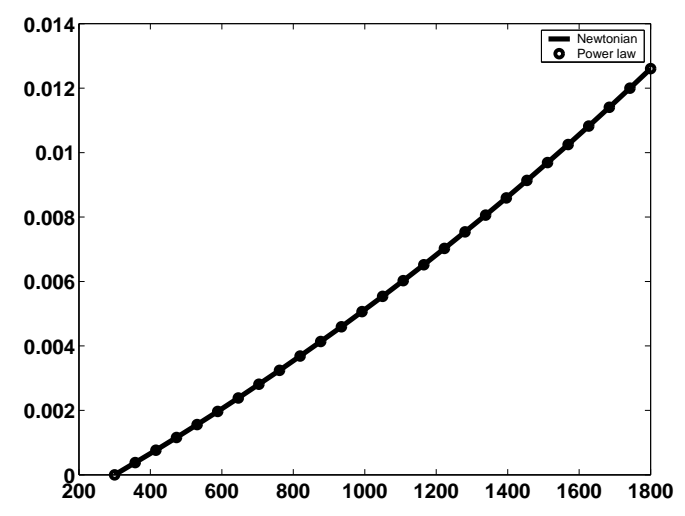

(b) Second $p$ - $A$ model

Figure 3: Convergence of the power law formulae (Equations 17 and 18) when $n=1.0$ to their corresponding Newtonian formulae (Equations 12 and 13) for elastic tubes (a) using the first $p$ - $A$ elastic model (Equation 1) with $\gamma=10^{5} \mathrm{~Pa}_{\mathrm{m}}{ }^{-2}$, $L=0.25 \mathrm{~m}, R_{o}=0.03 \mathrm{~m}, \mu=k=0.1$ Pa.s, and $p_{\text {ou }}=0.0 \mathrm{~Pa}$; and (b) using the second $p$ - $A$ elastic model (Equation 2) with $\beta=500$ Pa.m, $L=0.35 \mathrm{~m}$, $R_{o}=0.025 \mathrm{~m}, \mu=k=0.075$ Pa.s, and $p_{\text {ou }}=300 \mathrm{~Pa}$. The vertical axis in the sub-figures represents the volumetric flow rate, $Q$, in $\mathrm{m}^{3} \cdot \mathrm{s}^{-1}$ while the horizontal axis represents the inlet boundary pressure, $p_{i n}$, in $\mathrm{Pa}$. 
where $\alpha$ is the axial momentum flux correction factor which is related to the flow velocity profile, $\kappa$ is a viscosity friction coefficient, $\rho$ is the fluid mass density, and $A_{\text {in }}$ and $A_{\text {ou }}$ are the inlet and outlet cross sectional area of the tube respectively.

It is difficult to compare the newly derived formulae with the old ones which are obtained from the 1D Navier-Stokes model. First no comparison can be made for the power law fluids because the 1D Navier-Stokes model is restricted to Newtonian fluids. As for the Newtonian case, the two models are based on different formulation frameworks and hence it is difficult to make a fair comparison between the two. One major difficulty is that the 1D Navier-Stokes model contains several parameters $(\alpha$, $\kappa$ and $\rho$ ) which are absent in the newly derived formulae. In particular, $\alpha$ is strongly dependent on the flow velocity profile and hence can cause major discrepancy with the new formulae which are based essentially on a specific fluid-dependent velocity profile. In [10] it has been demonstrated that the 1D model can be very sensitive to the variation in its parameters. However, in certain cases the $1 \mathrm{D}$ model can have a very close match with the newly derived formulae by adjusting the 1D parameters. For demonstration purposes, we produced two examples, seen in Figure 4, where we compared the 1D formulae with the new formulae for the Newtonian flow using the two $p$ - $A$ relations. In these examples, we 'tuned' the $1 \mathrm{D}$ parameters to match the flow as predicted by the new formulae. The 1D model can also be tuned to match the prediction of the new formulae using sets of parameters other than those used in these examples.

One of the advantages of the newly derived formulae over the formulae of the 1D Navier-Stokes model is that the new formulae are simpler than the 1D ones as they contain less parameters. The many parameters of the 1D model, although may be a source of more diversity in the flow modeling, can reduce the predictive capability of the model due to the difficulty of obtaining accurate values for the model parameters and hence the model merit may be relegated to a descriptive state by 'tuning' the parameters to match the observed flow.

Finally, it should be remarked that the newly derived formulae can be used in modeling and simulating the flow in networks of interconnected elastic tubes by using the pore-scale network modeling approach as proposed in [11]. An obvious advantage of the use of the newly derived formulae over the $1 \mathrm{D}$ formulae in a pore-scale modeling scheme is that the new formulae can describe the flow of nonNewtonian fluids of power law type which the 1D Navier-Stokes model cannot do because of its restriction to the Newtonian fluids. Other types of non-Newtonian fluids with other types of distensibility models, such as viscoelastic or elastic with 


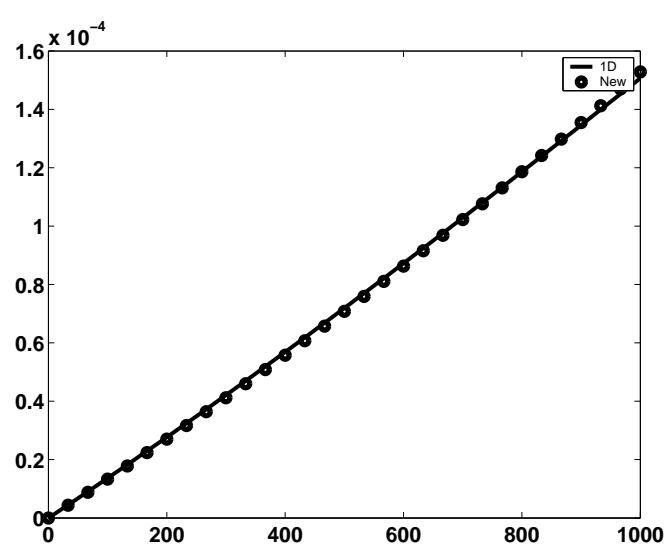

(a) First $p$ - $A$ model

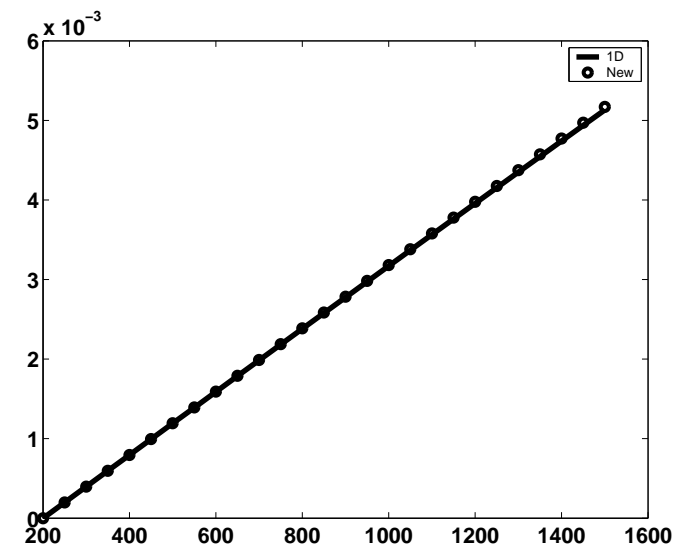

(b) Second $p$ - $A$ model

Figure 4: Comparing the 1D Navier-Stokes model formulae (Equations 19 and 20) with the newly derived formulae (Equations 12 and 13) for the flow of Newtonian fluids (a) using the first $p$ - $A$ elastic model (Equation 1) with $\alpha=1.345, \gamma=$ $2.0 \times 10^{7}$ Pa.m ${ }^{-2}, \mu=0.3$ Pa.s, $\rho=1000.0 \mathrm{~kg} . \mathrm{m}^{-3}, L=0.1 \mathrm{~m}, R_{o}=0.01 \mathrm{~m}$, and $p_{\text {ou }}=0.0 \mathrm{~Pa}$; and (b) using the second $p$ - $A$ elastic model (Equation 2) with $\alpha=1.333, \beta=5.0 \times 10^{5}$ Pa.m, $\mu=0.05$ Pa.s, $\rho=900.0$ kg.m ${ }^{-3}, L=0.1 \mathrm{~m}$, $R_{o}=0.015 \mathrm{~m}$, and $p_{\text {ou }}=200 \mathrm{~Pa}$. The vertical axis in the sub-figures represents the volumetric flow rate, $Q$, in $\mathrm{m}^{3} \cdot \mathrm{s}^{-1}$ while the horizontal axis represents the inlet boundary pressure, $p_{i n}$, in $\mathrm{Pa}$.

different $p$ - $A$ constitutive relations to those used in the present paper, can also be used in pore-scale modeling if pertinent analytical or empirical formulae were obtained.

\section{Conclusions}

In this paper we derived analytical formulae for the volumetric flow rate as a function of the two pressure boundary conditions, the fluid rheology, and the duct geometry for the flow of Newtonian and power law fluids in distensible tubes of regular cylindrical shapes. In these derivations we used two pressure-area constitutive relations of elastic nature. The method is based on a lubrication approximation where the flow velocity profile is assumed to be determined locally by the fluid rheology and the size of the local cross sectional area as in the case of the flow in a tube with a cross sectional area that, under flow state, is constant in shape and size over the whole tube length.

The Newtonian formulae can be used as an alternative to the previously derived formulae [17] which are based on the 1D Navier-Stokes flow in distensible tubes. 
As well as their possible use in modeling the flow in single distensible tubes, the derived expressions can be used in modeling the flow in elastic networks, as an alternative to the widely used 1D Navier-Stokes finite element method [18], in conjunction with the previously-proposed [11] pore-scale modeling approach. The newly derived power law formulae will facilitate modeling non-Newtonian rheology in single distensible tubes and networks of interconnected distensible tubes by the use, in the latter case, of pore-scale modeling. This is a major addition to the existing modeling capabilities which are limited to the Newtonian rheology.

For the purpose of validation, several sensible trends have been observed. These include (a) the convergence of the derived formulae to their corresponding rigid tube formulae with increasing the stiffness of the tube wall, and (b) the convergence of the power law formulae to their Newtonian equivalents when the power law index, $n$, is set to unity. Thorough tests have revealed that the newly derived formulae produce mathematically and physically sensible results in diverse situations of fluid rheology, tube geometry and boundary conditions.

A brief comparison has been made with the Newtonian formulae that were derived previously from the 1D Navier-Stokes distensible model. A major advantage of the newly derived formulae over the 1D Navier-Stokes formulae is the accommodation of the non-Newtonian rheology in the form of the power law model.

The derivation method proposed in this paper can in principle be extended to less regular geometries (e.g. converging-diverging) or to regular geometries but with non-cylindrical shape (e.g. square or elliptic cross section) although empirical or numerical, rather than analytical, approaches may be needed. Similarly, the method may also be extended to fluid rheologies other than Newtonian and power law fluids with possible restriction to the use of empirical or numerical, instead of analytical, approaches due to potential mathematical difficulties.

\section{Nomenclature}

\footnotetext{
$\alpha$ correction factor for axial momentum flux in the 1D model

$\beta$ stiffness factor in the second pressure-area model

$\gamma$ stiffness factor in the first pressure-area model

$\dot{\gamma}$ shear strain rate

$\kappa \quad$ viscosity friction coefficient
} 
$\mu \quad$ fluid dynamic viscosity

$\rho \quad$ fluid mass density

$\tau \quad$ shear stress

$A \quad$ tube cross sectional area corresponding to pressure $p$

$A_{\text {in }}$ tube cross sectional area at inlet

$A_{o} \quad$ tube reference cross sectional area corresponding to reference pressure

$A_{\text {ou }}$ tube cross sectional area at outlet

$k$ power law consistency factor

$L \quad$ tube length

$n$ power law flow behavior index

$p \quad$ axial pressure

$p_{\text {in }} \quad$ pressure at tube inlet

$p_{\text {ou }}$ pressure at tube outlet

$Q \quad$ volumetric flow rate

$r \quad$ tube radius

$R_{o} \quad$ tube radius corresponding to $A_{o}$

$x \quad$ tube axial coordinate

\section{References}

[1] A.C.L. Barnard; W.A. Hunt; W.P. Timlake; E. Varley. A Theory of Fluid Flow in Compliant Tubes. Biophysical Journal, 6(6):717-724, 1966.

[2] A.C.L. Barnard; W.A. Hunt; W.P. Timlake; E. Varley. Peaking of the Pressure Pulse in Fluid-Filled Tubes of Spatially Varying Compliance. Biophysical Journal, 6(6):735-746, 1966.

[3] M.S. Olufsen. A one-dimensional fluid dynamic model of the systemic arteries. Computational Modeling in Biological Fluid Dynamics: The IMA Volumes in Mathematics and its Applications, 124:167-187, 2001.

[4] N.P. Smith; A.J. Pullan; P.J. Hunter. An Anatomically Based Model of Transient Coronary Blood Flow in the Heart. SIAM Journal on Applied Mathematics, 62(3):990-1018, 2002.

[5] L. Formaggia; D. Lamponi; A. Quarteroni. One-dimensional models for blood flow in arteries. Journal of Engineering Mathematics, 47(3/4):251-276, 2003. 
[6] S.J. Sherwin; V. Franke; J. Peiró; K. Parker. One-dimensional modelling of a vascular network in space-time variables. Journal of Engineering Mathematics, 47(3-4):217-250, 2003.

[7] K. Vajravelu; S. Sreenadh; P. Devaki; K.V. Prasad. Mathematical model for a Herschel-Bulkley fluid flow in an elastic tube. Central European Journal of Physics, 9(5):1357-1365, 2011.

[8] P.K. Mandal. An unsteady analysis of non-Newtonian blood flow through tapered arteries with a stenosis. International Journal of Non-Linear Mechanics, 40(1):151-164, 2005.

[9] S. Sreedharamalle; D. Palluru; D. Reddy; Krishnaiah. Unsteady Flow of a Jeffrey Fluid In An Elastic Tube with A Stenosis. Proceedings of the International Conference on Fluid Dynamics and Thermodynamics Technologies, 33:136-142, 2012.

[10] T. Sochi. Comparing Poiseuille with 1D Navier-Stokes Flow in Rigid and Distensible Tubes and Networks. Submitted, 2013. arXiv:1305.2546.

[11] T. Sochi. Pore-Scale Modeling of Navier-Stokes Flow in Distensible Networks and Porous Media. Submitted, 2013. arXiv:1309.7568.

[12] L. Formaggia; F. Nobile; A. Quarteroni; A. Veneziani. Multiscale modelling of the circulatory system: a preliminary analysis. Computing and Visualization in Science, 2(3):75-83, 1999.

[13] J. Alastruey; S.M. Moore; K.H. Parker; T. David; J. Peiró S.J. Sherwin. Reduced modelling of blood flow in the cerebral circulation: Coupling 1-D, 0-D and cerebral auto-regulation models. International Journal for Numerical Methods in Fluids, 56(8):1061-1067, 2008.

[14] J. Janela; A.B. de Moura; A. Sequeira. Comparing Absorbing Boundary Conditions for a 3D Non Newtonian Fluid-Structure Interaction Model for Blood Flow in Arteries. Mecánica Computacional, XXIX(59):5961-5971, 2010.

[15] T. Sochi. Pore-Scale Modeling of Non-Newtonian Flow in Porous Media. PhD thesis, Imperial College London, 2007.

[16] T. Sochi. Using the Euler-Lagrange variational principle to obtain flow relations for generalized Newtonian fluids. Rheologica Acta, 53(1):15-22, 2014. 
[17] T. Sochi. Navier-Stokes Flow in Cylindrical Elastic Tubes. Journal of Applied Fluid Mechanics (Accepted).

[18] T. Sochi. One-Dimensional Navier-Stokes Finite Element Flow Model. Technical Report, 2013. arXiv:1304.2320. 